\title{
DEEP CONVOLUTIONAL NEURAL NETWORK WITH TENSORFLOW AND KERAS TO CLASSIFY SKIN CANCER IMAGES
}

\author{
HOUSSAM BENBRAHIM, HANAÂ HACHIMI ${ }^{*}$ AND AOUATIF AMINE ${ }^{\ddagger}$
}

\begin{abstract}
Skin cancer is a dangerous disease causing a high proportion of deaths around the world. Any diagnosis of cancer begins with a careful clinical examination, followed by a blood test and medical imaging examinations. Medical imaging is today one of the main tools for diagnosing cancers. It allows us to obtain precise images, internal organs and thus to visualize the possible tumours that they present. These images provide information on the location, size and evolutionary stage of tumour lesions. Automatic classification of skin tumours using images is an important task that can help doctors, laboratory technologists, and researchers to make the best decisions. This work has developed a classification model of skin tumours in images using Deep Learning with a Convolutional Neural Network based on TensorFlow and Keras model. This architecture is tested in the HAM10000 dataset consists of 10,015 dermatoscopic images. The results of the classification of the experiment show that the accuracy was achieved by our model, which is in order of $94.06 \%$ in the validation set and $93.93 \%$ in the test set.
\end{abstract}

Key words: Skin Cancer, Image Classification, Deep Learning, Convolutional Neural Network, TensorFlow, Keras, HAM10000 Dataset.

AMS subject classifications. 68U10, 68T05

1. Introduction. Skin cancer is the most frequently diagnosed disease in the world, it has an impact on the quality of life and it is can be deadly [1]. Skin cancer is considered as an important problem in public health, however, most cases are avoidable if detected early with a better prediction [2]. In Morocco, cancer is a health major problem requiring a comprehensive policy of care [3]. The situation in Morocco is very disturbing, there are approximately 30,000 new cases of cancer per year. Cancer is responsible for $7.2 \%$ of all deaths in Morocco, whereas skin cancer has been growing rapidly and it achieves ascending numbers of cases per year [4]. The diagnosis of skin cancer is necessary to reduce the negative effects of the dangerous development of this disease [5], which is usually performed with a doctor using visual inspection. This inspection is dependent on the experience and training of the doctor. In general, doctors can identify skin cancer with a sensitivity of $75 \%$ and specificity of $87 \%$ [6]. New techniques of image recognition such as Deep Learning technology can help doctors to better diagnose skin cancer disease. This method uses powerful and very advanced algorithms that achieve effective results [7]. Convolutional Neural Networks are the most frequently used Deep Learning algorithms, which are based on the human visual cortex. Its methods have been favourably admitted for numerous imaging classification [8]. In Morocco, the use of new technologies is very low in the health sector, which also negatively affects the detection of cancer in general and specifically skin cancer [9]. Morocco needs a national electronic system for the health sector based on powerful and advanced technologies [10], that can allow doctors to better analyze and diagnose all diseases and especially skin cancer. The general objective of this paper is to develop a system based on Deep Convolutional Neural Networks with TensorFlow and Keras that will be able to detect skin cancer. For this reason, this work used a test database named HAM10000, consists of 10,015 dermatoscopic images, and it has 7 different classes of skin cancer. The use of an intelligent model, which is based on powerful and innovative technologies, will be able to help doctors make a better diagnosis of skin cancer. This system

\footnotetext{
*BOSS-Team, GS-Laboratory, National School of Applied Sciences, Ibn Tofail University, Kenitra, Morocco (houssam. benbrahim@uit.ac.ma).

${ }^{\dagger}$ BOSS-Team, GS-Laboratory, National School of Applied Sciences, Ibn Tofail University, Kenitra, Morocco (hanaa. hachimi@univ-ibntofail.ac.ma).

${ }_{\ddagger}$ BOSS-Team, GS-Laboratory, National School of Applied Sciences, Ibn Tofail University, Kenitra, Morocco (aouatif. amine@uit.ac.ma).
} 
will aim to avoid the consequences of delay or bad diagnosis, as well as, to sensibilize Moroccan doctors for the importance and the necessity to employ its advanced techniques in medical imaging examinations.

2. Deep Learning. Deep Learning represents a class of Machine Learning and Artificial Intelligence (AI) techniques where the machine can learn on its own, unlike traditional programming where the program simply executes predetermined rules [11]. What is first the Machine Learning concept?

Machine Learning (ML) is an innovative technology that gives the possibility of computers to learn through experience [12]. In ML programming, the learning process is supervised and the programmer is the person responsible for extracting the characteristics of an image, for example [7]. With ML, computers need data to analyze and to make predictions or decisions by themselves. In recent decades, various ML techniques were developed to create algorithms that could learn and improve independently [13]. Deep Learning (DL) is, therefore, a set of automatic learning techniques where the machine learns to recognize patterns by training on qualified data models. The algorithms that make its operations possible use mathematical concepts based essentially on Artificial Neural Network (ANN) [14]. The advantage of DL is that the program itself extracts the features, which makes the treatment faster, and more accurate.

2.1. Performance. In the human brain, each neuron receives about 100,000 electrical signals from other neurons to detect for example an object, on this concept and more specifically on the ANN that based the DL [15]. This network is composed of several "layers" of neurons, each one receiving and interpreting the information of the previous layer. The higher the number of neurons is, the more the result is deep [16]. For a high level of accuracy, DL programs must have access to big amounts of training data and it uses powerful processing methods, that were difficult for programmers before the advent of Big Data and Cloud Computing technologies. With its two latest advanced techniques, DL programming has become fast, simple and very effective [17].

2.2. Application subjects. DL was applied to several problems over the last few years. It allows us to perform all kinds of tasks at incredible speed. There are many different applications of DL, here are some examples of using this technique [18].

- Health care: DL can detect symptoms of diseases such as cancer detection and drug discovery, relying on image data for example.

- Speech recognition: DL is used for various applications for speech recognition, such as Google Voice, Cortana, Amazon Echo, etc.

- Autonomous cars: DL is employed by researchers to detect white lines, pedestrians crossing, signs, other vehicles on the road, etc.

- Security: DL is applied for face recognition and video surveillance.

This is not an exhaustive list, but there are many other applications for example in natural language processing, customer relationship management, recommendation systems, and bioinformatics [19].

3. Convolutional Neural Network. A Convolutional Neural Network (CNN) is a type of ANN, in which the connection pattern between neurons is inspired by the animal's visual cortex. However, CNN's are specifically designed to process input images. It gets automatically and strongly the principal features to use in classifying images, that means it learns directly from samples [27]. Through several specific layers of sophisticated mathematical operations, CNN performs this type of automatic selection and classification of features. This technique has marked great importance on the DL committee in recent years especially in the field of image recognition or classification, machine vision projects, as well as skin cancer detection [28].

3.1. CNN for Classification. Image classification refers to the task of retrieving information classes from an image, which is one of the most principal tasks in computer vision and pattern recognition[29]. However, the task of choosing the best characteristics is enormously lengthy and is frequently unsuccessful. Also, increasing the features of other images types become big trouble [30]. CNN can solve an image classification problem by choosing features that could be the pixel location, the colour, object edges, or any other characteristics that could be taken from the images. This process is performed automatically by CNN. The better and more effective the feature sets extracted, the more accurate and efficient the image classification we can obtain. CNN's try to find a solution to this problem by employing more hidden layers [31]. To classify images and to construct a 


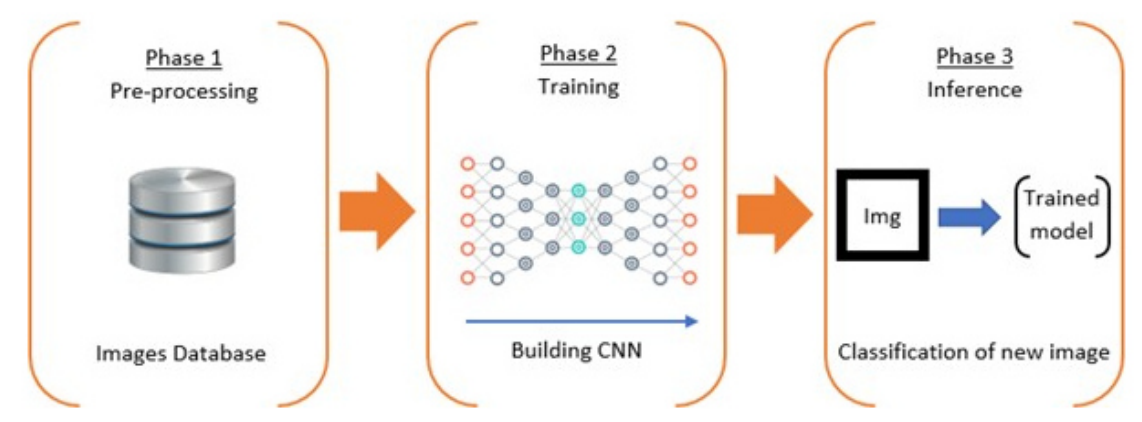

FIG. 3.1. The Deep Learning process.

digit identification system using CNN, the DL process consists of the following phases [32], the first step is the preprocessing of the input data, second, training the DL model, and finally the inference of the model. Firstly, the transformation of the images into a legible format. Secondly, the creation of a CNN and train it with several images. Finally, the validation of the model by the classification of new images. Figure 3.1 summarizes its phases.

3.2. CNN Architecture. In the training process, a deep neural network has multiple hidden layers, which are different in the type and their connectivity. CNN has multiple Convolutional layers, Non-Linearity layers, pooling layers, and fully connected layers [33]. The main purpose of the first layer is to apply a convolution operation to the input data, which means to detect different patterns or features from an input image, after that, it will transmit the result to the next layer. So, this layer defines a filter, which is also called a kernel. If the program slides the filter over the image and applies the dot product of the filter to the image pixels, the result would be a new image with all the edges. The second (ReLu layer) is integrated to replace all negative pixel values in the feature map by zero. The third layer is called Max-Pooling. This last integrates the outputs of a cluster of neurons in the precedent layer into one neuron in the next layer, which is an operation that finds the maximum values and simplifies the inputs. In other words, it reduces the number of parameters within the model. It turns the low-level data into higher-level information. And the fourth layer links every neuron in the precedents layer to every neurone in the next layer. Fully-Connected layers take the high-level filtered images from the previous layer and convert them into a vector [34].

4. TensorFlow. Created by the Google Brain team in 2011, TensorFlow is a dedicated system for tasks that require heavy numerical calculations. Originally called DistBelief, DistBelief's source code was changed and this tool became an application-based library. In 2015, it was renamed TensorFlow and Google made it open source. This system has an interface for Python and C++ and it went into version 1.0 in February 2017 [20]. Simply put, TensorFlow is a library of ML and deep neural networks, it is a toolbox for solving extremely complex mathematical problems. It can be conceived as a programming system in which the calculations are represented in the form of a data flow graph, which means we can create first a program and then execute it in a session [21]. TensorFlow offers several advantages for an application. It's able to run faster than pure Python code due to a $\mathrm{C} / \mathrm{C}++$ backend. It should be noted, that TensorFlow supports parallel computing, CPU and GPU $[22]$.

4.1. TensorFlow structure. TensorFlow's structure is built on the running of a data flow graph. This last has two basic units: the nodes and the edges. The first one represents a mathematical operation, and the second defines the multidimensional arrays, known as tensors. As cited before the standard use of this structure is to run a session after the creation of the graph. The session translates and passes the computations represented into the graphs to the desired environment to run them on a GPU or CPU [23]. Figure 4.1 represents a graph in a session with TensorFlow. For example, Z and Y are tensors. The program can call MatMul (an operation over the tensors Z and Y), after that, the operation Add is used, and the program can add the result with the tensor $\mathrm{X}$. To get the desired result $\mathrm{W}$, the outcoming tensors of each working pass through 


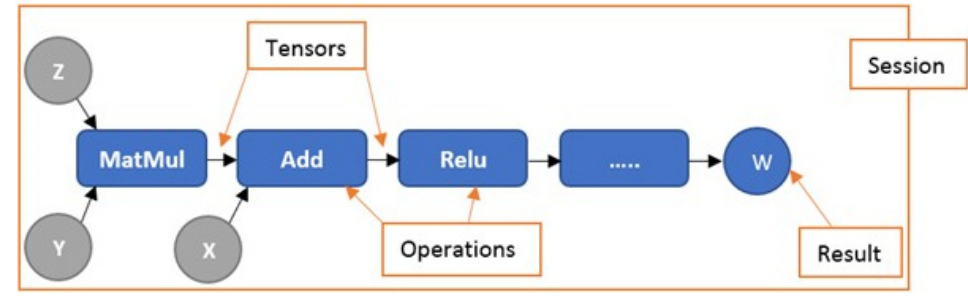

FIG. 4.1. TensorFlow work example.

TABLE 4.1

Tensor rank.

\begin{tabular}{|l|l|l|}
\hline Dimension & Mathematical Object & In Code \\
\hline Zero & Scalar & {$[1]$} \\
\hline One & Vector & {$[1,2,3,4, \ldots]$} \\
\hline Two & Matrix & {$[[1,2,3,4, \ldots],[1,2,3,4, \ldots],[1,2,3,4, \ldots], \ldots]$} \\
\hline Three & Tensor & {$[[[1,2, \ldots], \ldots],[[1,2, \ldots], \ldots],[[1,2, \ldots], \ldots], \ldots]$} \\
\hline
\end{tabular}

the next one up to the end. Finally, the program can build a session to execute the graph and implement the calculations. So, what means a tensor? In mathematics, more precisely in multilinear algebra and in differential geometry, a tensor designates a very general object whose value is expressed in a vector space. It can be used to represent multilinear applications or multivectors. A Tensor is a multidimensional array, it may be in different dimensions, zero-dimensional, one dimensional, 2-dimensional, etc. Table 4.1 summarize its information [24]. The Tensors giving programmers the freedom to form a dataset the way they want it, especially it's helpful when dealing with images, because of the nature of how the information contained in the images is encoded.

4.2. Architecture of TensorFlow. TensorFlow is built to support and execute computational graphs with new ML models and system-level optimization. TensorFlow supports programming language interfaces $\mathrm{C}++$, Python, and others. When we create and compile a program with the TensorFlow system, the language binding will invoke the TensorFlow library which includes the Core Execution System. It allowed programmers to deploy very complex computations on CPUs, GPUs, a desktop, server, or a mobile device. This signifies that programmers can build their program once, and then they can run it easily on different devices [25]. TensorFlow architecture has three primary steps. The first one is the data processing, programmers can write the codes in several programming languages, and then TensorFlow converts that into hardware instruction sets for CPU, GPU, Android, etc. The second one is to build the model in layers. The last one for training and estimating the model, for this reason, programmers can use Estimator and Keras model. The canned Estimator allows TensorFlow to support Neural Network, Logistic Regression, and Linear Regression [26].

5. Material and methods. In this study, a Deep CNN with Keras TensorFlow model to classify skin cancer images into seven classes was created, tested and validated. This work used the HAM10000 dataset that has seven several classes of skin cancer which are: actinic keratoses, basal cell carcinoma, benign keratosis-like lesions, dermatofibroma, melanoma, melanocytic nevi, and vascular lesions. In the test phase, the Anaconda environment (Python distribution) was used with TensorFlow and Keras model. The execution of our program is made on a CPU with Max RAM 16 GB. In this part, this paper presents a detailed description of the data set and it gives a general overview of the Keras TensorFlow model.

5.1. The HAM10000 dataset. The dataset "Human Against Machine with 10000 training images" is a great series of multi-source dermatoscopic images of common pigmented skin lesions [35]. Published and created by Philipp Tschandl (Medical University of Vienna), Cliff Rosendahl (University of Queensland) and Harald Kittler (Medical University of Vienna), Austria [36]. This database obtained from several populations acquired and stored by multiple modalities. The final dataset consists of 10,015 dermatoscopic images that are released as a training set for academic machine learning purposes and are publicly available through the ISIC archive. Response data are all encoded within a single CSV file (comma-separated value) file, with each 
TABle 5.1

Attributes information of the Dataset.

\begin{tabular}{|l|l|}
\hline Column name & Description \\
\hline lesion_id & Input image identifier of the form HAM___ \\
\hline image_id & Input image identifier of the form ISIC__ \\
\hline $\mathrm{dx}$ & 1. Actinic keratoses and intraepithelial carcinoma / Bowen's disease (akiec). \\
& 2. Basal cell carcinoma (bcc). \\
& 3. Benign keratosis-like lesions (solar lentigines /seborrheic keratoses and \\
& lichen-planus like ker- atoses, bkl). \\
& 4. Dermatofibroma (df). \\
& 5. Melanoma (mel). \\
& 7. Melanocytic nevi (nv). \\
\hline dx_type & 1. histo \\
& 2. follow_up \\
& 3. consensus \\
\hline age & The age of the patient \\
\hline sex & The gender of the patient \\
\hline localization & Location of the tumour in the patient's body \\
\hline
\end{tabular}

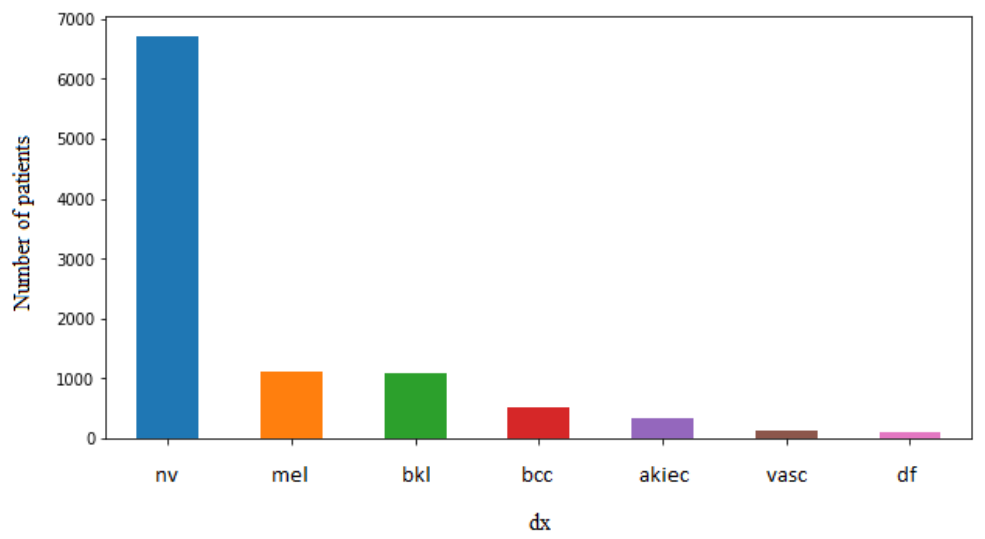

FIG. 6.1. The distribution of different classes of cell type.

classification response in a row. Table 5.1 shows the attribute information of the HAM10000 dataset.

5.2. Keras Model. Keras is the advanced model for the Python DL. Keras able to run on top of TensorFlow, Microsoft Cognitive, R, Toolkit, PlaidML, or Theano. It was created by François Chollet (Software Engineer at Google) to allow quick experiments. Its latest version is 2.3.1 (October 7, 2019). Keras is recommended for:

- Rapid and easy prototyping.

- Sustains convolutional networks and recurrent networks.

- Works on CPU and GPU.

For the test phase, the Keras sequential model is used by adding a list of layers instance to the constructor like the Convolutional layer, Pooling layer, Flatten and Dense layers.

\section{Results.}

6.1. Data Explore. After importing and cleaning the data, the exploratory data analysis was started. In this step, different attributes of the HAM10000 was explored. The database has seven different classes of cell type, Figure 6.1 visualizes its distribution. The maximum number of images are of type nv (6,705 images) and the minimum number is of type df (115 images), the others are classified as follows: mel 1,149 images, bkl 1,063 images, bcc 514 images, 327 images for akiec and 142 images for vasc. There are four categories of 


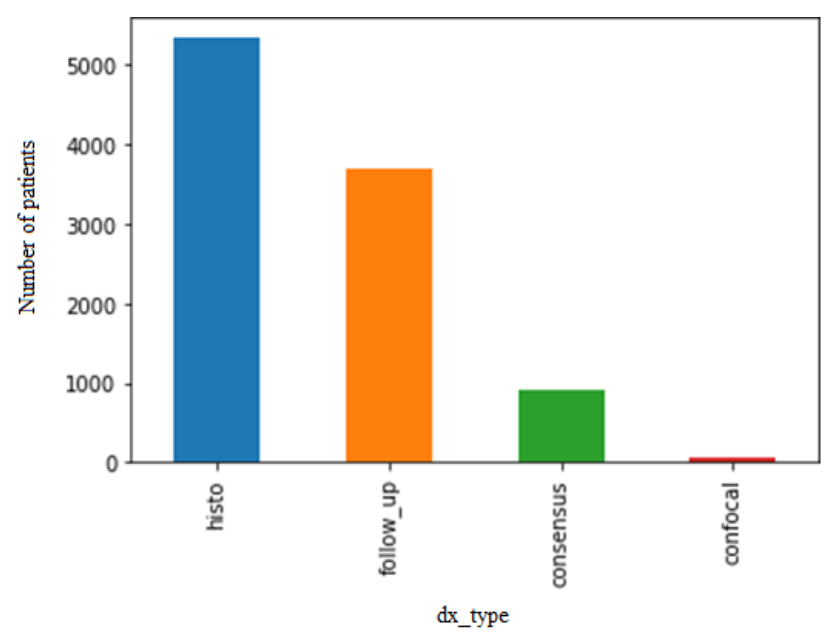

FIG. 6.2. The distribution of four categories of the dx_type.

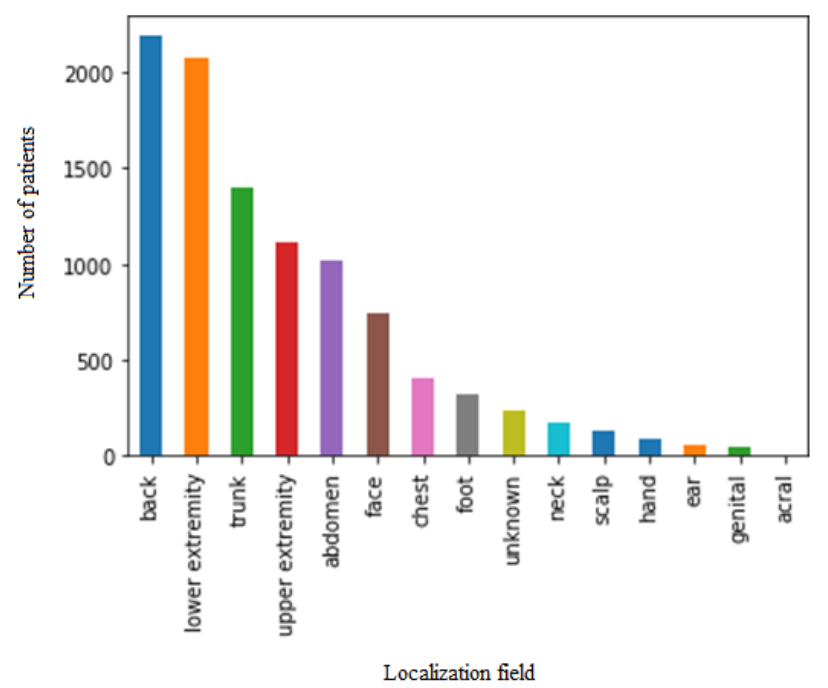

FIG. 6.3. The distribution of the localization field of skin cancer.

the $d x$ type which represent the technical validation field (ground truth), the distribution of this feature is plotted in Figure 6.2. More than 50\% of lesions were confirmed through histopathology (histo) which means diagnosis has been performed by specialized dermatopathologists. The rest (follow_up) is more than 3,500 instances, (consensus) for a loan of 1,000 instances, and finally confirmation by in-vivo confocal microscopy (confocal). Figure 6.3 represents the distribution of the localization field of skin cancer in the database. The results confirm that back, the lower extremity was heavily compromised regions of skin cancer at the first level, in the second level the trunk, upper extremity, abdomen and face have been marked, other cases continue to spread throughout the body. The age distribution shows that there are large cases of patients between 30 and 60 years. Figure 6.4 illustrates the results. For the gender of patients, the results affirm that more than $50 \%$ are male, the distribution of males and females is illustrated in Figure 6.5.

6.2. Experiments. In this phase, before the implementation of the model, the program loaded the images. The input data are dermoscopic lesion images in JPEG format where there are 7 possible disease categories. Figure 6.6 presents one sample for each disease category. The reel size of the loaded images is 450x600x3, which 


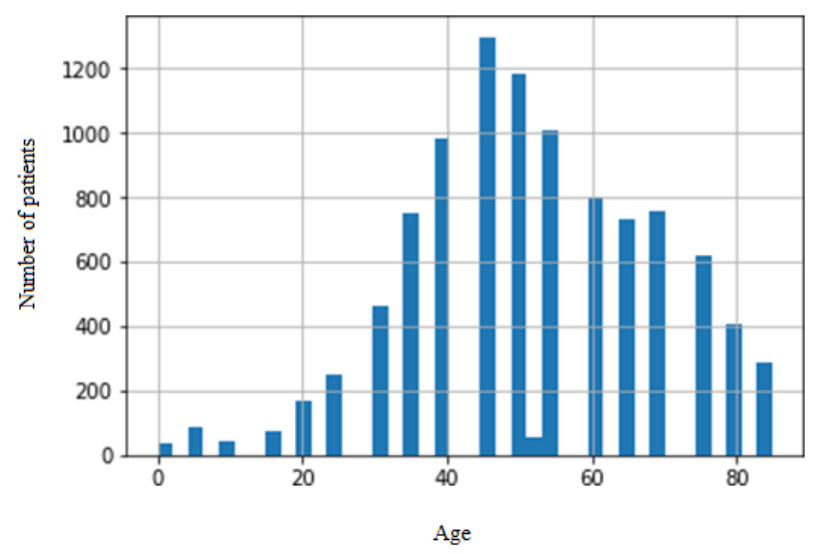

FIG. 6.4. The age distribution.

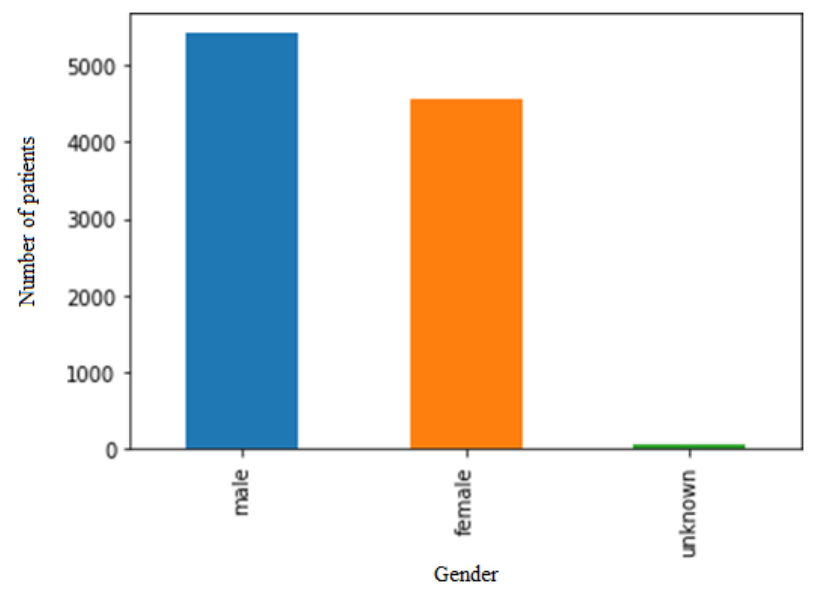

FIG. 6.5. The sex distribution.
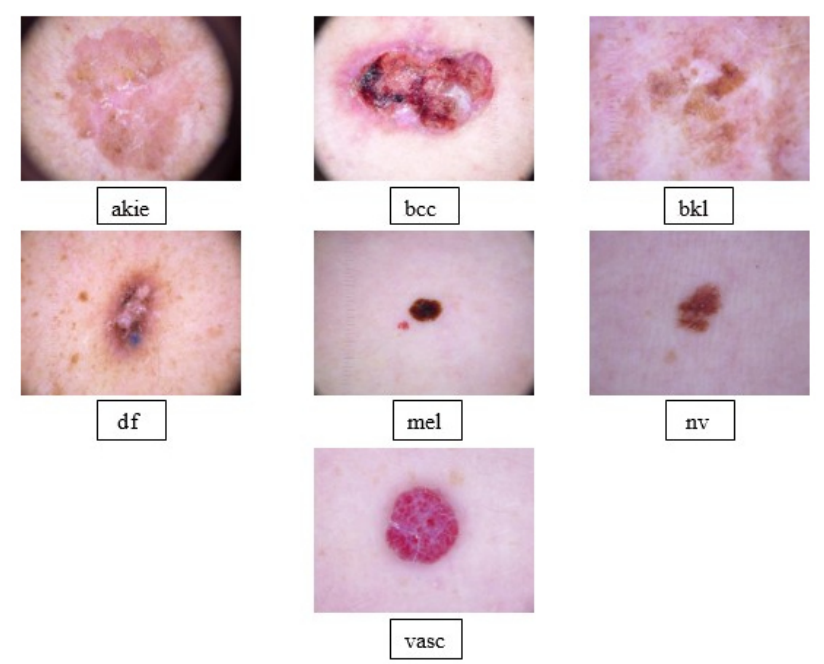

FIG. 6.6. An example of each skin cancer disease. 


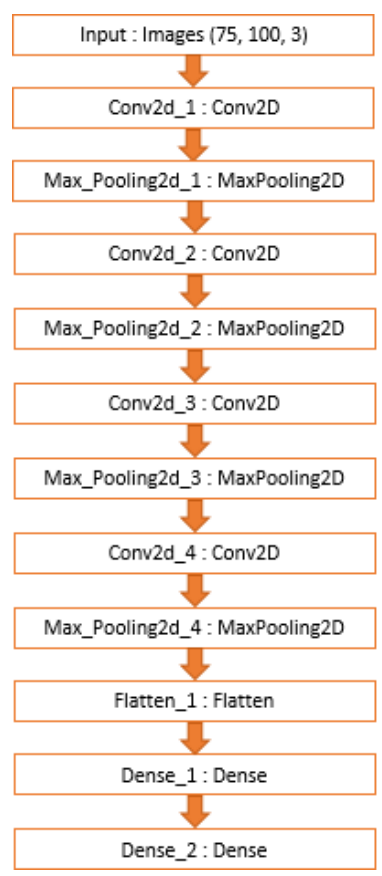

FIG. 6.7. The general architecture of our model.

TABLE 6.1

Model summary.

\begin{tabular}{|l|l|l|}
\hline Layer (type) & Output Shape & Param \# \\
\hline conv2d_1 (Conv2D) & (None, 75, 100, 64) & 1792 \\
\hline max_pooling2d_1 (MaxPooling2D) (Conv2D) & (None, 36, 49, 64) & 0 \\
\hline conv2d_2(Conv2D) & (None, 34, 47, 64) & 36928 \\
\hline max_pooling2d_2 (MaxPooling2D) & (None, 17, 23, 64) & 0 \\
\hline conv2d_3(Conv2D) & (None, 15, 21, 128) & 73856 \\
\hline max_pooling2d_3 (MaxPooling2D) & (None, 7, 10, 128) & 0 \\
\hline conv2d_4(Conv2D) & (None, 5, 8, 128) & 147584 \\
\hline max_pooling2d_4 (MaxPooling2D) & (None, 2, 4, 128) & 0 \\
\hline flatten (Flatten) & (None, 1024) & 0 \\
\hline dropout (Dropout) & (None, 1024) & 0 \\
\hline dense_1 (Dense) & (None, 512) & 524800 \\
\hline dense_2 (Dense) & (None, 7) & 3591 \\
\hline
\end{tabular}

TensorFlow can't handle, that's why the program resize all the images of the database HAM10000 (10,015 images) into $75 \times 100 \times 3$. For the implementation of the model, the database was partitioned into 3 sets ( $80 \%$ train, $10 \%$ validation and $10 \%$ test). In this step, the model receives $75 \times 100$ images as input, then has a sequence of four convolutional and pooling layers as feature extractors, followed by a flatten operation and two dense layers. A plot of the model is created in Figure 6.7. In the model, the first imported layer is the convolutional (Conv2D), the program uses 64 filters for the two firsts ones and 128 for the two last ones. Its layers are employed to extract features from the images. The second employed layer in the architecture is the pooling (MaxPool2D). This last is used four times to reduce the dimensionality of the images. After that, the program added a Flatten layer to transform the two-dimensional matrix of features into a vector. Also, the Dropout layer integrated to improve overfit on neural networks. Finally, the program integrated the Dense layer that regroups the results and generates a prediction. The CNN architecture is detailed in Table 6.1. In the first step, the performance of the model is calculated by measuring the accuracy index, it refers to the proximity of the measurements to giving value. Figures 6.8 shows the model accuracy of the architecture. Secondly, 


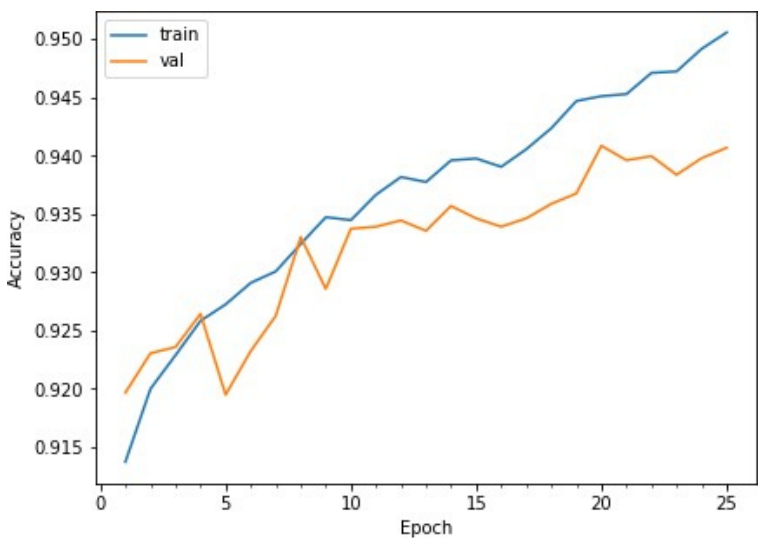

FIG. 6.8. Model accuracy.

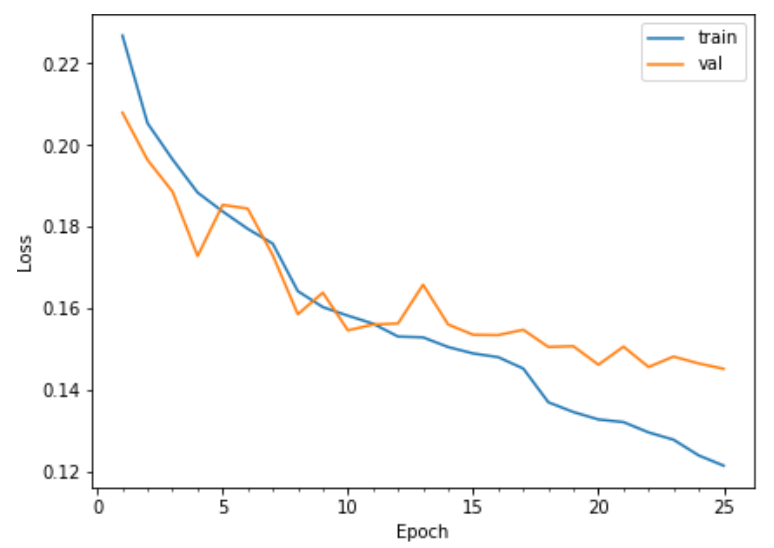

FIG. 6.9. Model loss.

establishing the model loss, It defines how much the modelling of the problem, which is an approximation of reality, loses information compared to the reality observed through the example data. Figure 6.9 plots the model loss of the architecture. This work confirms that the model achieves the accuracy of $94.06 \%$ in the validation set and $93.93 \%$ in the test set, as well as $14.52 \%$ for loss validation and $14.63 \%$ for test loss with epochs equal to 25. Epoch means an instant of time marked by an event that begins a new period, it is usually characterized by a distinctive development. These results show the importance of automatic image classification and especially the detection of skin cancer using its advanced technologies such as Deep Learning and more precisely CNN, TensorFlow, and Keras model. Morocco needs to use its techniques in the field of health in general and that Moroccan doctors in addition to their experiences and their training can be familiar with its approaches, methods, and techniques to increase the rate of a best and effective diagnosis.

7. Discussion. The overall objective of this work is to create an automatic classification of skin cancer images, for accurate identification of the tumour class. Fast, efficient and accurate detection of this disease can positively affect the next steps of the diagnosis. In this paper, a method based on deep CNNs with TensorFlow and Keras model was proposed for the extraction of the skin cancer in JPEG images. In this article, the input of the architecture is 75x100 images and the output is a layer of seven neurons because there are seven classes of skin cancer in the HAM10000 database. In this model, four layers of convolutions were created each with MaxPooling layer. Before feeding into a Dense layer the Dropout is a little trick to improve the efficiency of a neural network by throwing away some of the neurons. All of this is programmed in a sequential model based on TensorFlow with Keras. The experimental results proved that the proposed method can reach a very 
high accuracy of $94.06 \%$ in the validation set and $93.93 \%$ in the test set with epochs equal to 25 . In a similar study created by [37], the authors developed a classification of images from the HAM10000 dataset, they used convolution, centring and full connection layers in the model created with the VGGNET architecture. In their research, the test phase of the educated model, class validation was obtained at $85.62 \%$. In another work [38], the authors have trained a CNN based on the ResNet50 architecture to classify dermoscopy images of skin lesions. With their custom model, they obtained a balanced accuracy of $91 \%$ on the validation dataset. In [39], the authors created a skins cancer identification system of HAMl0000 dataset using CNN, the accuracy of training and testing of skins cancer identification system was $80 \%$ and $78 \%$. In [40], the authors proposed an automated system for skin lesion classification through transfer learning-based deep neural network (DCNN). The experimental results achieved an accuracy of $89.8 \%$. After all that we can say that the model proposed has achieved very important results and the architecture is succeed to classify dermoscopic images of skin lesions in one of the seven categories.

8. Conclusion. In this study, an architecture on Deep CNN using TensorFlow framework and Keras model was developed to classify 7 types of skin cancer. The implementation of the model was realized by applying an image classification structure on HAM10000 Database. The latter contains a significant number of skin cancer images in JPEG format knowing that there are seven possible disease classes. In Morocco, the number of cases of skin cancers is growing, and it can become dangerous due to a delay in the diagnosis phase or the failing prediction of the disease. The goal is to build a solution that able to classify skin tumours in the images. This application can help doctors, especially in Morocco to better diagnose, detect and quickly identify attacked patients by skin cancer and speed up the workflow. In this work, we design a CNN sequential model to classify the seven skin diseases. The results of the classification experiment show that the accuracy of the model was achieved, which had, in its best configuration, $94.06 \%$ and $93.93 \%$ in the validation set and test set, respectively. These results can convince doctors in Morocco to share digital images of their patients in private mode to create a common database that can be used as a reference for scientific research in DL context, also to enhance the use of advanced technologies for doctors. Our future work aims to implement the architecture proposed in this work into a Big Data environment, to prove the feasibility, the reliability of this model, and to improve their performance with several techniques like the use of Tensorflow on Spark.

\section{REFERENCES}

[1] R. L. Siegel, K. D. Miller, and A. Jemal, Cancer statistics, 2015, CA: a cancer journal for clinicians, 2015, vol. 65, no 1, pp. 5-29.

[2] C. WiLD, World cancer report, World Health Organization, 2014, pp. 482-494.

[3] R. BekKali, Lutte Contre Le Cancer Au Maroc- L'apport De La Fondation Lalla Salma, International Journal of Medicine and Surgery, 2017, vol. 4, no 1, pp. 55-59.

[4] Ministry of Health in Morocco and Lalla Salma Association of Fight Against Cancer, Plan national de prévention 2010-2019 Axes Stratégiques et Mesures, 2009.

[5] M. Harte And G. KNePil, Skin cancer detection, British dental journal 227, 2019, pp. 539-539.

[6] Y. A. Glickman, O. Filo, M. David, A. Yayon, M. Topaz, B. Zamir, A. Ginzburg, D. Rozenman, and G. Kenan, Electrical impedance scanning: a new approach to skin cancer diagnosis, Skin Research and Technology, 2003, vol. 9, no 3, pp. 262-268.

[7] L. Deng, And D. Yu, Deep learning: methods and applications, Foundations and Trends in Signal Processing, 2014, vol. 7, no 3-4, pp. 197-387.

[8] R. Zhao, W. Ouyang, H. Li, and X. Wang, Saliency detection by multi-context deep learning, in Proceedings of the IEEE Conference on Computer Vision and Pattern Recognition, 2015, pp. 1265-1274.

[9] H. Benbrahim, H. Hachimi, and A. Amine, Survey on the Use of Health Information Systems in Morocco: Current Situation, Obstacles and Proposed Solutions, in : International Conference on Advanced Intelligent Systems for Sustainable Development, Springer, Cham, 2018, pp. 197-204.

[10] H. Benbrahim, H. Hachimi, and A. Amine, Moroccan Electronic Health Record System, in: International Conference on Industrial Engineering and Operations Management, 2018, Paris, France.

[11] Y. Bengio, A. Courville, and P. Vincent, Representation learning: A review and new perspectives, IEEE transactions on pattern analysis and machine intelligence, 2013, vol. 35, no 8, pp. 1798-1828.

[12] S. Tanwar, Q. Bhatia, P. Patel, A. Kumari, P. K. Singh, and W. C. Hong, Machine learning adoption in blockchain-based smart applications: The challenges, and a way forward, IEEE Access, 2019, vol. 8, pp. 474-488.

[13] C. M. Bishop, Representation learning: Pattern recognition and machine learning, springer, 2006.

[14] J. Schmidhuber, Deep learning in neural networks: An overview, Neural networks, 2015, vol. 61, pp. 85-117. 
[15] S.A. Kalogirou, Applications of artificial neural-networks for energy systems, Applied energy, 2000, vol. 67, no 1-2, pp. 17-35.

[16] J. G. Lee, S. Jun, Y. W. Cho, H. Lee, G. B. Kim, J. B. Seo, and N. Kim, Deep learning in medical imaging: general overview, Korean journal of radiology, 2017, vol. 18, no 4, pp. 570-584.

[17] Q. Zhang, L.T. Yang, Z. Chen, And P. Li, A survey on deep learning for big data, Information Fusion, 2018, vol. 42, pp. 146-157.

[18] M. M. Najafabadi, F. Villanustre, T. M. Khoshgoftaar, N. Seliya, R. Wald, and E. Muharemagic, Deep learning applications and challenges in big data analytics, Journal of Big Data, 2015, vol. 2, no 1, pp. 1.

[19] N. F. Hordri, S. S. Yuhaniz, and S. M. Shamsuddin, Deep learning and its applications: a review, in : Conference on Postgraduate Annual Research on Informatics Seminar, 2016.

[20] M. Abadi, A. Agarwal, P. Barham, E. Brevdo, Z. Chen, C. Citro, G. S Corrado, A. Davis, J. Dean, M. DEvin, AND OTHERs, Tensorflow: Large-scale machine learning on heterogeneous distributed systems, arXiv preprint arXiv:1603.04467, 2016.

[21] M. Abadi, P. Barham, and J. Chen, Z. Chen, A. Davis, J. Dean, M. Devin, S. Ghemawat, G. Irving, M. Isard, and OTHERS, Tensorflow: A system for large-scale machine learning, in : 12th USENIX Symposium on Operating Systems Design and Implementation (OSDI 16), 2016, pp. 265-283.

[22] M. T. Martinez, An Overview of Google's Machine Intelligence Software TensorFlow, Sandia National Lab.(SNL-NM), Albuquerque, NM (United States), 2016.

[23] G. ZACCONe, Getting Started with TensorFlow, Packt Publishing Ltd, 2016.

[24] R. Bonnin, Building Machine Learning Projects with TensorFlow, Packt Publishing Ltd, 2016.

[25] P. Goldsborough, A tour of tensorflow, arXiv preprint arXiv:1610.01178, 2016.

[26] G. Zaccone, M.R. Karim, and A. Menshawy, Deep Learning with TensorFlow, Packt Publishing Ltd, 2017.

[27] Y. Kıм, Convolutional neural networks for sentence classification, arXiv preprint arXiv:1408.5882, 2014.

[28] A. Khan, A. Sohail, U. Zahoora, and A. S. Qureshi, A survey of the recent architectures of deep convolutional neural networks, arXiv preprint arXiv:1901.06032, 2019.

[29] W. Wang, D. Liang, Q. Chen, Y. Imamoto, X. H. Han, Q. Zhang, H. Hu, L. Lin, and Y. W. Chen, Medical Image Classification Using Deep Learning, in : Deep Learning in Healthcare, Springer, Cham, 2020, pp. 33-51.

[30] W. RAWAT, AND Z. WANG, Deep convolutional neural networks for image classification: A comprehensive review, Neural computation, 2017, vol. 29, no 9, pp. 2352-2449.

[31] Q. Li, W. Cai, X. Wang, Y. Zhou, , D. D. Feng, and M. Chen, Medical image classification with convolutional neural network, in 2014 13th international conference on control automation robotics \& vision (ICARCV). IEEE, 2014. pp. 844-848.

[32] I. Arel, D. C Rose, And T. P. Karnowski, Deep machine learning-a new frontier in artificial intelligence research [research frontier], IEEE computational intelligence magazine, 2010, vol. 5, no 4, pp. 13-18.

[33] A. HidAka AND T. KuRita, Consecutive dimensionality reduction by canonical correlation analysis for visualization of convolutional neural networks, in Proceedings of the ISCIE International Symposium on Stochastic Systems Theory and its Applications, the ISCIE Symposium on Stochastic Systems Theory and Its Applications, 2017. pp. 160-167.

[34] R. Yamashita, M. Nishio, R. K. G. Do, and K. Togashi, Convolutional neural networks: an overview and application in radiology, Insights into imaging, 2018, vol. 9, no 4, pp. 611-629.

[35] P. Tschande, C. Rosendahl, and H. Kittler, The HAM10000 dataset, a large collection of multi-source dermatoscopic images of common pigmented skin lesions, Scientific data, 2018, vol. 5, pp. 180161.

[36] N. Codella, V. Rotemberg, P. Tschandl, M. E. Celebi, S. Dusza, D. Gutman, B. Helba, A. Kalloo, K. Liopyris, M. Marchetti, AND others, Skin lesion analysis toward melanoma detection 2018: A challenge hosted by the international skin imaging collaboration (isic), arXiv preprint arXiv:1902.03368, 2019.

[37] E. Çevik And K. Zengin, Classification of Skin Lesions in Dermatoscopic Images with Deep Convolution Network, Avrupa Bilim ve Teknoloji Dergisi, 2019, pp. 309-318.

[38] N. D. Reddy, Classification of Dermoscopy Images using Deep Learning, arXiv preprint arXiv:1808.01607, 2018.

[39] A. A. Nugroho, I. Slamet, and Sugiyanto, Skins cancer identification system of HAMl0000 skin cancer dataset using convolutional neural network, in : AIP Conference Proceedings, AIP Publishing LLC, 2019, pp. 020039.

[40] M. A. Khan, M. Y. Javed, M. Sharif, T. Saba, and A. Rehman, Multi-model deep neural network based features extraction and optimal selection approach for skin lesion classification, in : 2019 international conference on computer and information sciences (ICCIS), IEEE, 2019, pp. 1-7.

Edited by: Anand Nayyar

Received: Mar 31, 2020

Accepted: Jul 17, 2020 